Published in final edited form as:

Radiother Oncol. 2019 March ; 132: 55-62. doi:10.1016/j.radonc.2018.11.002.

\title{
Phase I Trial of Concurrent Stereotactic Body Radiotherapy and Nelfinavir for Locally Advanced Borderline or Unresectable Pancreatic Adenocarcinoma
}

\author{
Chi Lin, MD PhD ${ }^{1, \dagger}$, Vivek Verma, MD², Quan P. Ly, MD³, Audrey Lazenby, MD ${ }^{4}$, Aaron \\ Sasson, MD ${ }^{5}$, James K. Schwarz, $\mathbf{M D}^{6}$, Jane L. Meza, PhD $^{7}$, Chandrakanth Are, MD ${ }^{3}$, \\ Sicong Li, PhD ${ }^{1}$, Shuo Wang, PhD ${ }^{1}$, Stephen M. Hahn, MD ${ }^{8}$, and Jean L. Grem, MD \\ ${ }^{1}$ Department of Radiation Oncology, University of Nebraska Medical Center, Omaha, NE, USA \\ 2Department of Radiation Oncology, Allegheny General Hospital, Pittsburgh, PA, USA \\ ${ }^{3}$ Department of Surgery, University of Nebraska Medical Center, Omaha, NE, USA \\ ${ }^{4}$ Department of Pathology, University of Nebraska Medical Center, Omaha, NE, USA \\ ${ }^{5}$ Department of Surgery, Stony Brook School of Medicine, Stony Brook, NY, USA \\ ${ }^{6}$ Department of Internal Medicine, Division of Hematology Oncology, University of Nebraska \\ Medical Center, Omaha, NE, USA \\ ${ }^{7}$ Department of Biostatistics, University of Nebraska Medical Center, Omaha, NE, USA \\ ${ }^{8}$ Department of Radiation Oncology, The University of Texas MD Anderson Cancer Center, \\ Houston, TX
}

\section{Abstract}

INTRODUCTION: The HIV protease inhibitor nelfinavir (NFV) displays notable radiosensitizing effects. There have been no studies evaluating combined stereotactic body radiotherapy (SBRT) and NFV for borderline/unresectable pancreatic cancer. The primary objective of this phase I trial (NCT01068327) was to determine the maximum tolerated SBRT/NFV dose, and secondarily evaluate outcomes.

METHODS: Following initial imaging, pathologic confirmation, and staging laparoscopy, subjects initially received three 3-week cycles of gemcitabine/leucovorin/fluorouracil; patients without radiologic progression received 5-fraction SBRT/NFV. Dose escalation was as follows: 1) 25 Gy/625 mg BID x3wks; 2) 25 Gy/1250 mg BID x3wks; 3) 30 Gy/1250 mg BID x3wks; 4) 35

\footnotetext{
${ }^{\dagger}$ Corresponding Author: Chi Lin, MD PhD, Department of Radiation Oncology, Buffett Cancer Center, $505 \mathrm{~S} 45^{\text {th }}$ Street, Omaha, NE 68106, Phone: 402-552-3844, Fax: 402-552-3443, clin@unmc.edu.

Publisher's Disclaimer: This is a PDF file of an unedited manuscript that has been accepted for publication. As a service to our customers we are providing this early version of the manuscript. The manuscript will undergo copyediting, typesetting, and review of the resulting proof before it is published in its final citable form. Please note that during the production process errors may be discovered which could affect the content, and all legal disclaimers that apply to the journal pertain.

Disclaimers: A portion of this work was presented at the 2011 Annual Meeting of the American Society for Radiation Oncology and 2014 Annual Meeting of the American Society of Clinical Oncology.

Conflicts of Interest: All authors declare no conflicts of interest.

All authors declare that conflicts of interest do not exist.
} 
Gy/1250 mg BID x3wks; 5) 35 Gy/1250 mg BID x5wks; and 6) 40 Gy/1250 mg BID x5wks. Pancreaticoduodenectomy was performed thereafter if resectable; if not, gemcitabine/leucovorin/ fluorouracil was administered.

RESULTS: Forty-six patients enrolled (10/2008-5/2013); 39 received protocol-directed therapy. Sixteen (41\%) experienced any grade $\geq 2$ event during and 1 month after SBRT. Four grade 3 and both grade 4 events occurred in a single patient at the initial dose level. $40 \mathrm{~Gy} / 1250 \mathrm{mg}$ BID $\mathrm{x} 5 \mathrm{wks}$ was the maximum tolerated dose. Five patients had late gastrointestinal bleeding $(\mathrm{n}=2$ superior mesenteric artery pseudoaneurysm, $\mathrm{n}=1$ disease progression, $\mathrm{n}=1$ lower tract, $\mathrm{n}=1$ unknown location). The median overall survival was 14.4 months. Six (15\%) patients recurred locally; median local failure-free survival was not reached. The median distant failure-free survival was 11 months, and median all failure-free survival was 10 months.

CONCLUSIONS: Concurrent SBRT (40 Gy)/NFV (1250 mg BID) for locally advanced pancreatic cancer is feasible and safe, although careful attention to treatment planning parameters is recommended to reduce the incidence of late gastrointestinal bleeding.

\section{Keywords}

pancreatic cancer; pancreas; stereotactic body radiation therapy; nelfinavir

\section{INTRODUCTION}

Neoadjuvant chemoradiation therapy is an attractive approach to manage locally advanced pancreatic cancer (LAPC) [1]. Not only can it allow patients with borderline resectable (BR) or unresectable (UR) disease to undergo resection, it may also reduce the rate of positive margins [2]. Selecting non-progressors in a more refined manner may better avoid surgical morbidities [3-5]. It is also more favorable in light of the notable disadvantages of adjuvant therapy, such as suboptimal receipt of chemoradiation owing to surgical complications as well as the postoperative hypoxic environment [6].

Additionally, neoadjuvant treatment better favors the delivery of stereotactic body radiation therapy (SBRT), also known as stereotactic ablative radiotherapy, which is a highly conformal modality that affords low doses to surrounding organs-at-risk [7-8]. It has the major advantages of avoiding a protracted interval from diagnosis to surgery, along with increased patient convenience and enhanced cost-effectiveness [9].

Numerous prospective trials have reported the safety and efficacy of neoadjuvant SBRT for LAPC, particularly when delivered with 3-6 fractions [10]. However, tumor control and response rates remain relatively low. To improve upon these outcomes, dose-escalation is theoretically possible but often comes at the cost of greater toxicities; another approach is to administer radiosensitizer(s) in efforts to improve the therapeutic ratio. The human immunodeficiency virus (HIV) protease inhibitor NFV (NFV) interferes with PI3K-Akt signaling, which is normally involved in cellular growth and metabolism [11]. Multiple preclinical studies have demonstrated radiosensitizing effects, including in pancreatic cancer cells [12-14] and those that specifically received single-fraction ablative (8 Gy) radiotherapy (RT) [13]. Based on prospective trials in HIV patients, it is tolerated well [15]; moreover, 
many prospective investigations of other neoplasms have illustrated encouraging safety and efficacy of NFV with RT [16-21].

To date, there has been one phase I [22] and phase II [23] study evaluating NFV in LAPC, which displayed satisfactory results. However, both these studies utilized conventionallyfractionated RT without ablative dosing. Because the fractional doses in SBRT are equivalent or higher than those achievable by conventional fractionation, there could be incremental improvements in durable tumor control or potentially even survival [24]; the addition of NFV in such settings may be even more pronounced. This is the first known study of combined NFV and SBRT for LAPC; the primary objective of this phase I trial was to assess the safety of this regimen and secondarily evaluate outcomes.

\section{MATERIALS \& METHODS}

\section{Study Participants}

This was an Institutional Review Board approved phase I trial of concurrent NFV and SBRT for BR or UR LAPC (Clinicaltrials.gov number NCT01068327). Eligible patients were $\geq 19$ years of age with newly-diagnosed, pathologically-confirmed BR or UR (as defined by the National Comprehensive Cancer Network [25]) non-metastatic pancreatic adenocarcinoma not exceeding $8 \mathrm{~cm}$ in maximal dimension. Additional criteria were Karnofsky performance status of $\Varangle 60 \%$ and serum laboratory values appropriate to receive chemotherapy. Patients with biliary or gastroduodenal obstruction were allowed if drainage or surgical bypass had been performed prior to starting SBRT. Patients with prior malignancy were also allowed, provided therapy was completed $>5$ years prior to enrollment and there was no clinical evidence of that malignancy. Exclusion criteria were patients who had received prior RT to the area (defined as disease overlap with the $20 \%$ isodose line), uncontrolled intercurrent illnesses (such as history of significant bowel pathology, including bleeding/ulcers), HIV infection, allergies to chemotherapy or antiemetics, inability to provide informed consent, or pregnant/nursing women. Additionally, patients receiving medications known to be contraindicated with NFV, along with those not able to undergo staging laparoscopy and fiducial marker implantation for any reason, were also considered ineligible.

\section{Study Procedures}

All patients underwent initial workup by a medical, surgical, and radiation oncologist with a complete history and physical examination. Blood counts and chemistries along with CA 19-9 level were obtained, in addition to pathologic diagnosis and imaging (contrastenhanced 3 phases, i.e. arterial, venous, and delayed phase computed tomography (CT), magnetic resonance imaging (MRI), and/or positron emission tomography $(n=20)$ ). Patients then underwent staging laparoscopy to confirm lack of metastatic peritoneal disease, along with placement of two fiducial markers (Cook Medical, platinum embolization coil, $0.45 \times 5$ $\mathrm{mm}$ ) in the pancreas around the mass approximately two centimeters apart. An proton pump inhibitor was started at the time of SBRT and continued for 1 month.

Patients initially received three cycles of 3-week chemotherapy with intravenous gemcitabine $750 \mathrm{mg} / \mathrm{m}^{2}$ (females) or $900 \mathrm{mg} / \mathrm{m}^{2}$ (males) over 30 minutes on days 1 and 8 , 
calcium leucovorin $50 \mathrm{mg} / \mathrm{m}^{2}$ over 30 minutes on days 1 and 8 , and 5-fluorouracil 2700 $\mathrm{mg} / \mathrm{m}^{2}$ over 24 hours on days 1 and 8 . Restaging CT and/or MRI were subsequently performed; patients were removed from the study in case of disease progression (defined per the Response Evaluation Criteria in Solid Tumors, or distant metastatic disease).

Provided there was no progressive disease, patients then started on oral NFV for two weeks prior to initiating SBRT. Pharmacokinetic studies confirmed appropriate concentrations of plasma NFV in each patient [26]. The dose escalation schema for this study was as follows: 1) 25 Gy + 625 mg BID NFV for 3 weeks; 2) 25 Gy + 1250 mg BID NFV for 3 weeks; 3 ) 30 Gy + 1250 mg BID NFV for 3 weeks; 4) 35 Gy + 1250 mg BID NFV for 3 weeks; 5) 35 Gy $+1250 \mathrm{mg}$ BID NFV for 5 weeks; and 6) $40 \mathrm{~Gy}+1250 \mathrm{mg}$ BID NFV for 5 weeks. The standard $3+3$ dose-escalation design was used; however, if the previous patient had not been clinically evaluated for at least one month, the next patient was enrolled on the same dose level. Twenty patients were enrolled at the highest dose level to most completely assess safety and efficacy, three of which were removed from protocol therapy following discovery of progressive/distant disease.

SBRT treatment planning consisted of a four-dimensional CT simulation (4DCT, 2-3 mm slice thickness) with custom immobilization devices (e.g. BodyFIX ${ }^{\mathrm{TM}}$, Elekta, Stockholm, Sweden) and intravenous/oral contrast. Target volume delineation was performed in accordance with the International Commission on Radiation Units and Measurements Report 50. Elective nodal irradiation was not performed [27]. The gross tumor volume $\left(\mathrm{GTV}_{\text {primary }}\right.$ and $\left.\mathrm{GTV}_{\text {nodes }}(\geq \mathrm{cm})\right)$ was contoured using a combination of all available information on CT, MRI, and/or PET/CT imaging, along with clinical and endoscopic information when available. The integrated clinical tumor volume (CTV) was constructed from the aforementioned GTVs, additionally contouring the areas between them, along with additional margins obtained from 4DCT information to compensate for internal organ motion. The planning target volume (PTV) provided a $6 \mathrm{~mm}$ margin around the integrated CTV to compensate for variability in treatment setup, with exception at the gastric and duodenal interfaces (adjusted to avoid overlap with these areas). The prescribed doses ranged from 5-8 Gy $\times 5$ daily fractions (corresponding to biologically effective doses of 37.5 Gy, $48 \mathrm{~Gy}, 59.5 \mathrm{~Gy}$, and $72 \mathrm{~Gy}$ ); although the prescribed dose was to cover at least $95 \%$ of the PTV, 93\% was acceptable for technically challenging circumstances related to bowel dose constraints. The recommended maximum and median doses were $110 \%$ and $100 \%$, respectively. SBRT doses took into account tissue inhomogeneity corrections. Organs at risk were contoured in accordance with Radiation Therapy Oncology Group (RTOG) guidelines [28]. Dose constraints were from previous studies [27, 29-32], SBRT dose tolerance publications [33], and RTOG 0631 [34]. Of note, no more than $5 \mathrm{cc}$ of the stomach/small bowel was allowed to receive higher than $32 \mathrm{~Gy}$. Owing to the balance between bowel dose and PTV coverage, however, additional duodenal dose constraints could be utilized Supplementary Table 1). The percent of total kidney volume (sum of the left and right kidney volumes) receiving 15 Gy (3 Gy per fraction) was required to be less than $35 \%$ of the total kidney volume. The maximum dose to any point within the spinal cord was not allowed to exceed $15 \mathrm{~Gy}$ ( $3 \mathrm{~Gy}$ per fraction). At least $700 \mathrm{ml}$ or $35 \%$ of the normal liver was required to receive a total dose less than 15 Gy (3 Gy per fraction). SBRT was delivered on a Novalis linear accelerator with a M3 multileaf collimator and daily image guidance using ExacTrac 
orthogonal kilovoltage imaging (BrainLAB, Heimstetten, Germany). Because the breathing patterns were adequate in all patients, respiratory-gated delivery was used.

\section{Follow-Up}

While on therapy, patients were seen weekly by a medical and radiation oncologist, with toxicity assessment per the Common Terminology Criteria for Adverse Events (CTCAE) version 3.0. Patients underwent repeat $\mathrm{CT}$ and/or MRI imaging at 4 weeks following SBRT completion to evaluate for resectability at the surgical oncologist's discretion; if considered resectable, pancreaticoduodenectomy or partial pancreatectomy was performed 5-8 weeks following SBRT completion, followed by 3 more cycles of 3-week adjuvant gemcitabine/ leucovorin/fluorouracil. If unresectable, gemcitabine/leucovorin/fluorouracil was delivered indefinitely pending tolerance and patient/physician preference. The chemotherapy regimen was selected from results of an institutional phase I protocol [35]. In either case, patients underwent surveillance contrast-enhanced CT and CA 19-9 every three months thereafter.

\section{Pathologic Response}

For patients who underwent surgical resection, pathological response of the tumor to neoadjuvant therapy was re-evaluated by an experienced gastrointestinal pathologist. The pathological response score was assigned from 0-9, which was generated from the following three categories (tumor gland dropout, tumor shrinkage, and global tumor kill). Tumor gland dropout was scored as the following: 0 ) $>90 \%$ of tumor nests back-to-back (minimal dropout, densely spaced), 1) 50-90\% of tumor nests back-to-back (mild dropout), 2) 10-49\% of tumor nests back-to-back (moderate dropout), and 3) $<10 \%$ of tumor nests back-to-back (marked dropout, hypodense). Tumor shrinkage was quantified as follows: 0) $>90 \%$ tumor cells with abundant cytoplasm, large multicellular glands (not shrunken), 1) 50-90\% tumor cells with abundant cytoplasm, large multicellular glands (mildly shrunken), 2) 10-49\% tumor cells with abundant cytoplasm, large multicellular glands (moderately shrunken) and 3) $<10 \%$ tumor cells with abundant cytoplasm, large multicellular glands (markedly shrunken). Lastly, global tumor kill was defined as follows: 0) No definite response (poor to no response), 1) minimal response (some fibrosis, moderate amount of cancer present), 2) moderate response (minimal residual cancer present) and 3) complete response (no residual cancer identified).

\section{Statistical Analysis}

The primary endpoint of this trial was to evaluate safety and toxicity profiles. A doselimiting toxicity (DLT) was defined as 1) CTCAE grade $\_4$ hematologic toxicity, 2) CTCAE grade $\geq 3$ nonhematologic toxicity except grade $\geq 4$ nausea/vomiting/diarrhea not controlled with medical treatment, or 3) a $\geq 1$ week break from SBRT that was possibly, probably, or definitely related to SBRT/NFV. Occurrence of a DLT required entry of three additional patients to the particular dose level at which it occurred; if two instances in the same dose level were experienced, de-escalation to the prior dose level was obligated. The maximum tolerated dose (MTD) of NFV/SBRT was defined as the highest dose level at which no greater than one DLT was experienced. Secondary endpoints included analysis of local (infield, including in-field primary and nodal disease, if applicable) failures by overlaying the area of suspected recurrence with the SBRT field; all other relapses were categorized as 
distant (out-of-field). Consequently, distant failure-free survival (DFFS), (overall) failurefree survival (FFS), and overall survival (OS) were computed actuarially by the KaplanMeier method and compared with the log-rank test. Local failures were evaluated in cumulative incidence format using competing risk methodology. All statistics were performed with SAS version 9.4 (Cary, NC) and denoted $\mathrm{p}<0.05$ as statistically significant.

\section{RESULTS}

Figure 1 demonstrates a flow diagram of the trial. Altogether, 46 patients consented to enroll (all but one (involving the pancreatic body) had disease in the pancreatic head). Seven patients were taken off trial prior to undergoing SBRT/NFV, with the most common reason being disease progression (of these seven patients, one did not receive any therapy, three received 1 cycle and three completed 3 cycles of induction chemotherapy). Thus, 39 patients underwent SBRT/NFV per protocol and were subsequently analyzed (Table 1).

Toxicity assessment is detailed in Table 2 . There were 21, 11, and 2 instances of grades 2, 3, and 4 toxicities, respectively. Of the 39 patients, $16(41 \%)$ patients experienced any grade $\geq 2$ adverse event. Of note, all grade $\geq 3$ adverse events had started prior to receiving concurrent SBRT/NFV. Four instances of grade 3 events, plus both grade 4 events, occurred in a single patient at the initial dose level. This patient developed a liver abscess that necessitated surgical drainage and intravenous antibiotics. Although these adverse events were possibly worse after SBRT/NFV, this was not considered directly caused by SBRT/NFV, given that all started prior to receiving SBRT/NFV. Of note, there were no statistical differences in any toxicities or grade $\geq 3$ events based on NFV dosing or in patients receiving 5-6 Gy/fraction as compared to 7-8 Gy/fraction ( $p>0.05$ for both). Thus, the highest dose level (40 Gy in 5 fractions) tested in this investigation was considered the MTD (Supplementary Table 2).

The median follow-up was 14.4 months (range, 1.0-45.7); all patients had passed away at the time of data analysis. Figure 2 illustrates outcomes following SBRT/NFV. Six (15\%) patients experienced any local failure. The median DFFS was 11 (95\% confidence interval (CI), 7-15) months, and most distant metastases occurred in the liver, peritoneum, and/or lung. The median FFS was 10 (95\% CI, 6-14) months. The median OS in all patients was 14 (95\% CI, 12-18) months. Most patients died from disease, but five patients died from late gastrointestinal (GI) bleeding ( $\mathrm{n}=2$ superior mesenteric artery pseudoaneurysm (bleeding was stopped after SMA stent placement in both cases, but both families chose to pursue comfort care measures), $\mathrm{n}=1$ unknown location (patient refused esophagogastroduodenoscopy (EGD)), n=1 lower GI location with negative EGD, n=1 disease progression invading the GI tract on EGD). Thus, aside from the patient with clear EGD evidence of bleeding from disease progression, the remainder four may have possibly experienced late grade 5 events. However, although treatment effect can never be entirely excluded, it was the consensus of the multidisciplinary management team that one of these patients had a lower GI bleed with negative EGD, which could be from lower dose spillage to other portions of the bowel, but would not be as likely as areas exposed to higher dose. Another patient had bleeding of an unknown location, but had undergone pancreaticoduodenectomy (and thus removal of the area exposed to high dose). 
To further explore patients that developed GI bleeding, a dosimetric analysis was performed between four patients that experienced bleeding (having excluded one patient whose bleeding was unequivocally visualized secondary to disease progression) and 23 patients without bleeding who received 35-40 Gy (Table 3). Although no values were statistically significant, there were trends towards significance in multiple parameters, most notably duodenal V35, V20, and V10.

After completing SBRT/NFV, 10 patients were found to have metastases ( 4 by imaging, 6 by exploratory surgery), 15 were deemed to have unresectable disease ( 6 by imaging, 9 had attempted pancreaticoduodenectomy), and 1 had declining performance status. Thirteen patients were thus considered resectable after therapy; 1 refused surgery and therefore twelve underwent pancreaticoduodenectomy (eleven with negative margins and six with positive nodes). Of these 12 patients, 9 were initially borderline resectable and 4 unresectable; all but one received 7-8 Gy per fraction. There was no 30-day postoperative mortality.

Of the 12 resected patients, 1 had no response, 6 had Evans grade IIa response (10-50\%), 4 had Evans grade IIb (50-90\%) response, and 1 had a complete response (Supplementary Table 3). Linear regression analysis of the pathological response score and combined SBRT/NFV dose revealed a positive correlation with a slope of 0.24 (Supplementary Figure $1)$.

Figure 3 shows Kaplan-Meier OS curves of patients that received surgery versus lack thereof (Figure 3A), along with a comparison of the 5-6 Gy and 7-8 Gy cohorts for patients with unresectable disease (Figure 3B). The median OS for those undergoing surgery was 19 (95\% CI, 12-30) months, which was statistically similar to unresected patients (median OS 14 months, 95\% CI 8-24 months) ( $\mathrm{p}=0.11$ ). In the unresected cohort, as compared to those receiving 5-6 Gy (median OS 10 months, 95\% CI 4-13 months), there was higher OS in whom 7-8 Gy (median OS 18 months, 95\% CI 12-24 months) was delivered ( $\mathrm{p}=0.002$ ). Local failures in these cohorts could not be reliably compared owing to the relatively few events.

\section{DISCUSSION}

Novel radiosensitizers such as NFV may be advantageous when combined with ablative RT for LAPC, but there are no such reports to date. The results of this phase I trial demonstrate that this approach is well tolerated, including at the maximum dose level (40 Gy in 5 fractions combined with 5 weeks of $1250 \mathrm{mg}$ twice daily NFV). However, late gastrointestinal bleeding was found to occur herein, which must be further addressed. Therefore, further prospective investigations utilizing SBRT/NFV are recommended to follow-up these results.

Evaluating the safety and efficacy of SBRT regimens utilizing 7-8 Gy/fraction is important, especially in the presence of a candidate radiosensitizer. This is primarily because 5-6 Gy/ fraction dosing yields biologically effective doses (BEDs) that are similar to palliative $(<45-50 \mathrm{~Gy})$ conventionally-fractionated regimens, with similar results [36]. Although this 
study was not powered to compare outcomes, it is hence not surprising that there was a trend towards improved OS in unresected patients receiving 7-8 Gy/fraction over 5-6 Gy/fraction. Although OS (which can be influenced by a multitude of factors) is acknowledged as a suboptimal endpoint for this comparison as opposed to local recurrence, it was notable that relatively few patients had local recurrences herein; whether the presence of NFV was a causative factor cannot be addressed by this nonrandomized study. Nevertheless, the local control herein compares favorably to an existing phase II trial showing 1-year freedom from local progression of $78 \%$ (2-year value of $83 \%$ in this study) [37]. Although that trial used $33 \mathrm{~Gy}$ in 5 fractions, there are other currently ongoing trials seeking to escalate doses up to $60 \mathrm{~Gy}$ in 5 fractions [38].

Another option that may prove advantageous to improving the therapeutic ratio is simultaneous integrated boosting (SIB), especially because there is evidence for improved outcomes with dose-escalated therapy (albeit in the conventionally-fractionated setting) [39]. A "threshold" of $\sim 100$ Gy has been posited to achieve optimal and durable tumor control, but has not been well-validated for pancreatic cancer [36]. Because this threshold corresponds to a 5 -fraction total dose of $50 \mathrm{~Gy}$, the potential utility of a radiosensitizer to possibly decrease this "required threshold" would be noteworthy if eventually proven.

The proportion of initially BR and UR patients able to undergo resection, along with R0 resection rates, in this trial was also comparable or numerically higher than those of many prospective trials [1]. To this extent, it is noteworthy that all but two patients able to undergo pancreaticoduodenectomy received 7-8 Gy/fraction. Although this may indicate that doseescalated SBRT can better address vascular-abutting disease, meaningful statistical comparisons (e.g. local recurrence) cannot be performed owing to the low sample sizes. SBRT dose-escalation to vascular areas can also be performed by means of SIB to the tumor-vessel interface, which is a frequent area of positive margins at time of resection [40].

Our outcomes with 7-8 Gy/fraction SBRT and NFV (median OS 18 months) are numerically higher than existing data [37] and similar to those of the prospective ARCII study for LAPC (17 months) [23], which consisted of concurrent NFV and conventionally-fractionated (50.4 Gy, boosted to $59.4 \mathrm{~Gy}$ ). Although our FFS of 10 months was nearly double the progressionfree survival of 5.5 months in that study, there are many confounding factors that preclude direct comparison, including definition and measurement of recurrent/progressive disease. However, our acute toxicity outcomes roughly appear comparable or even numerically favorable to those data. Although this could be related to utilizing a stereotactic technique, that study performed some degree of elective nodal irradiation without further nuances of treatment planning reported.

The finding of five patients dying from late gastrointestinal (GI) bleeding must be qualified. We first acknowledge that the occurrence of superior mesenteric artery pseudo-aneurysms $(n=2)$ are characteristic of radiation-induced vascular injury, as seen in several other neoplasms [41-51]. Indeed, using these examples, carefully measuring doses to arterial structures may be of benefit, especially in the setting of high doses per fraction. Whether normal tissue complications depend on the caliber of the particular blood vessel remains an ongoing issue, however. Nevertheless, assuming that the patient with EGD evidence of 
bleeding from progressive disease as well as the subject with EGD-negative lower GI bleed were highly unlikely related to SBRT/NFV, the rate of $8 \%$ (3/39 patients) herein may be higher than the work of Herman and colleagues [37] (4\%, 2/47 patients). Although NFV cannot be ruled out as an associated factor, it is important to contextualize that the conebeam computed tomographic image guidance in that study was likely superior to the orthogonal kilovoltage imaging herein. Additionally, that trial prescribed 33 Gy over 5 fractions, whereas most (72\%) patients in this trial received 35-40 Gy over 5 fractions; of the five patients that experienced bleeding herein, four received $40 \mathrm{~Gy}$ and the other $35 \mathrm{~Gy}$. Despite the lower sample sizes, there were trends towards statistical significance of multiple dosimetric parameters and bleeding. Hence, we endorse careful consideration of stricter constraints as well as measuring other parameters such as doses to the superior mesenteric vessels. Our current recommendation for the GI tract constaint is that no more than $0.5 \mathrm{cc}$ of the stomach/small bowel receives higher than $32 \mathrm{~Gy}$. With this constraint, we have treated over 20 patients with 40 Gy in 5 fractions in institutional phase II trials without further late GI bleeding.

There are multiple possible sources reducing applicability of these data in addition to those mentioned above. The prophylactic use of a proton inhibitor and selection of patients without significant bowel/ulcerative disease should be noted, along with the individualized judgment regarding PTV margins and target coverage versus bowel doses. Next, as other work has pointed out, there is an inherent difficulty in evaluating acute versus late toxicities owing to the nature of follow-up as well as death [37,52], which is why adverse events were reported as an aggregate, similar to other investigations [23]. Additionally, subgroup evaluation of survival by dose per fraction is largely a posthoc analysis with nonrobust sample sizes. Lastly, the individual effect of NFV on any assessed endpoint cannot be ascertained owing to the non-randomized nature of this prospective trial.

\section{Supplementary Material}

Refer to Web version on PubMed Central for supplementary material.

\section{Acknowledgements:}

The authors thank Mary Kos and Eugene Sehi for their assistance with this trial.

Funding: This study was in part supported by Spore Grant Number 2 P50 CA127297-06A1.

\section{REFERENCES}

1. Verma V, Li J, Lin C. Neoadjuvant therapy for pancreatic cancer: systematic review of postoperative morbidity, mortality, and complications. Am J Clin Oncol 2016;39:302-313. [PubMed: 26950464]

2. Gillen S, Schuster T, Meyer Zum Buschenfelde C, Friess H, Kleeff J. Preoperative/neoadjuvant therapy in pancreatic cancer: a systematic review and meta-analysis of response and resection percentages. PLoS Med 2010;7:e1000267. [PubMed: 20422030]

3. Raut CP, Evans DB, Crane CH, Pisters PW, Wolff RA. Neoadjuvant therapy for resectable pancreatic cancer. Surg Oncol Clin N Am 2004;13:639-661. [PubMed: 15350939]

4. Wayne JD, Abdalla EK, Wolff RA, et al. Localized adenocarcinoma of the pancreas: the rationale for preoperative chemoradiation. Oncologist 2002;7:34-45. 
5. Sohn TA, Yeo CJ, Cameron JL, et al. Resected adenocarcinoma of the pancreas-616 patients: results, outcomes, and prognostic indicators. J Gastroenterol Surg 2000;4:567-579.

6. Lim KH, Chung E, Khan A, et al. Neoadjuvant therapy of pancreatic cancer: the emerging paradigm? Oncologist 2012;17:192-200. [PubMed: 22250057]

7. Verma V, Lazenby AJ, Zheng D, et al. Dosimetric parameters correlate with duodenal histopathologic damage after stereotactic body radiotherapy for pancreatic cancer: Secondary analysis of a prospective clinical trial. Radiother Oncol 2017;122:464-469. [PubMed: 28089484]

8. Verma V, Bhirud AR, Denniston KA, Bennion NR, Lin C. Quantification of renal function following stereotactic body radiotherapy for pancreatic cancer: secondary dosimetric analysis of a prospective clinical trial. Radiat Oncol 2017;12:71. [PubMed: 28449702]

9. Murphy JD, Chang DT, Abelson J, et al. Cost-effectiveness of modern radiotherapy techniques in locally advanced pancreatic cancer. Cancer 2012;118:1119-1129. [PubMed: 21773972]

10. Petrelli F, Comito T, Ghidini A, et al. Stereotactic Body Radiation Therapy for Locally Advanced Pancreatic Cancer: A Systematic Review and Pooled Analysis of 19 Trials. Int J Radiat Oncol Biol Phys 2017;97:313-322. [PubMed: 28068239]

11. Bae SS, Cho H, Mu J, Birnbaum MJ. Isoform-specific regulation of insulin-dependent glucose uptake by Akt/protein kinase B. J Biol Chem 2003;278:49530-49536. [PubMed: 14522993]

12. Gupta AK, Cerniglia GJ, Mick R, McKenna WG, Muschel RJ. HIV protease inhibitors block Akt signaling and radiosensitize tumor cells both in vitro and in vivo. Cancer Res 2005;65:8256-8265. [PubMed: 16166302]

13. Pore N, Gupta AK, Cerniglia GJ, et al. Nelfinavir down-regulates hypoxia-inducible factor 1alpha and VEGF expression and increases tumor oxygenation: implications for radiotherapy. Cancer Res 2006;66:9252-9259. [PubMed: 16982770]

14. Al-Assar O, Bittner MI, Lunardi S, et al. The radiosensitizing effects of Nelfinavir on pancreatic cancer with and without pancreatic stellate cells. Radiother Oncol 2016;119:300-305. [PubMed: 27247056]

15. Saag MS, Tabas P, Sension M, et al. Randomized, double-blind comparison of two nelfinavir doses plus nucleosides in HIV-infected patients (Agouron study 511). AIDS 2001;15:1971-1978. [PubMed: 11600825]

16. Rengan R, Mick R, Pryma D, et al. A Phase I Trial of the HIV Protease Inhibitor Nelfinavir with Concurrent Chemoradiotherapy (CT-RT) for Unresectable Stage IIIA/IIIB NSCLC: A Report of Toxicities and Clinical Response. J Thorac Oncol 2012;7:709-715. [PubMed: 22425919]

17. Buijsen J, Lammering G, Jansen RL, et al. Phase I trial of the combination of the Akt inhibitor nelfinavir and chemoradiation for locally advanced rectal cancer. Radiother Oncol 2013;107:184188. [PubMed: 23647753]

18. Alonso-Basanta M, Fang P, Maity A, et al. A phase I study of nelfinavir concurrent with temozolomide and radiotherapy in patients with glioblastoma multiforme. J Neurooncol 2014;116:365-372. [PubMed: 24194293]

19. Blumenthal GM, Gills JJ, Ballas MS, et al. A phase I trial of the HIV protease inhibitor nelfinavir in adults with solid tumors. Oncotarget 2014;5:8161-8172. [PubMed: 25327558]

20. Hoover AC, Milhem MM, Anderson CM, et al. Efficacy of nelfinavir as monotherapy in refractory adenoid cystic carcinoma: Results of a phase II clinical trial. Head Neck 2015;37:722-726. [PubMed: 24596143]

21. Hill EJ, Roberts C, Franklin JM, et al. Clinical Trial of Oral Nelfinavir before and during Radiation Therapy for Advanced Rectal Cancer. Clin Cancer Res 2016;22:1922-1931. [PubMed: 26861457]

22. Brunner TB, Geiger M, Grabenbauer GG, et al. Phase I trial of the human immunodeficiency virus protease inhibitor nelfinavir and chemoradiation for locally advanced pancreatic cancer. J Clin Oncol 2008;26:2699-2706. [PubMed: 18509182]

23. Wilson JM, Fokas E, Dutton SJ, et al. ARCII: A phase II trial of the HIV protease inhibitor Nelfinavir in combination with chemoradiation for locally advanced inoperable pancreatic cancer. Radiother Oncol 2016;119:306-311. [PubMed: 27117177]

24. Zhong J, Patel K, Switchenko J, et al. Outcomes for patients with locally advanced pancreatic adenocarcinoma treated with stereotactic body radiation therapy versus conventionally fractionated radiation. Cancer 2017;123:3486-3493. [PubMed: 28493288] 
25. National Comprehensive Cancer Network. Pancreatic adenocarcinoma. Version 3.2017 https:// www.nccn.org/professionals/physician_gls/pdf/pancreatic.pdf Accessed March 27, 2018.

26. Kattel K, Evande R, Tan C, et al. Impact of CYP2C19 polymorphism on the pharmacokinetics of nelfinavir in patients with pancreatic cancer. Br J Clin Pharmacol 2015;80:267-275. [PubMed: 25752914]

27. Chang DT, Schellenberg D, Shen J, et al. Stereotactic radiotherapy for unresectable adenocarcinoma of the pancreas. Cancer 2009;115:665-672. [PubMed: 19117351]

28. Jabbour SK, Hashem SA, Bosch W, et al. Upper abdominal normal organ contouring guidelines and atlas: a Radiation Therapy Oncology Group consensus. Pract Radiat Oncol 2014;4:82-89. [PubMed: 24890348]

29. Koong AC, Le QT, Ho A, et al. Phase I study of stereotactic radiosurgery in patients with locally advanced pancreatic cancer. Int J Radiat Oncol Biol Phys 2004;58:1017-1021. [PubMed: 15001240]

30. Chuong MD, Springett GM, Freilich JM, et al. Stereotactic body radiation therapy for locally advanced and borderline resectable pancreatic cancer is effective and well-tolerated. Int J Radiat Oncol Biol Phys 2013;86:516-522. [PubMed: 23562768]

31. Su TS, Liang P, Lu HZ, et al. Stereotactic body radiotherapy using CyberKnife for locally advanced unresectable and metastatic pancreatic cancer. World J Gastroenterol 2015;21:81568162. [PubMed: 26185389]

32. Mellon EA, Hoffe SE, Springett GM, et al. Long-term outcomes of induction chemotherapy and neoadjuvant stereotactic body radiotherapy for borderline resectable and locally advanced pancreatic adenocarcinoma. Acta Oncol 2015;54:979-985. [PubMed: 25734581]

33. Grimm J, LaCouture T, Croce R, et al. Dose tolerance limits and dose volume histogram evaluation for stereotactic body radiotherapy. J Appl Clin Med Phys 2011;12:267-292.

34. Radiation Therapy Oncology Group. RTOG 0631 Protocol Information. https://www.rtog.org/ ClinicalTrials/ProtocolTable/StudyDetails.aspx?study=0631 Accessed January 28, 2018.

35. Grem JL, Quinn MG, Keith B, et al. Phase I trial of weekly gemcitabine in combination with infusional 5-fluorodeoxyuridine and oral calcium leucovorin in adult cancer patients. Cancer Chemoth and Pharmacol 2003;53:487-496.

36. Crane $\mathrm{CH}$. Hypofractionated ablative radiotherapy for locally advanced pancreatic cancer. J Radiat Res 2016;57:i53-i57. [PubMed: 27029741]

37. Herman JM, Chang DT, Goodman KA, et al. Phase 2 multi-institutional trial evaluating gemcitabine and stereotactic body radiotherapy for patients with locally advanced unresectable pancreatic adenocarcinoma. Cancer 2015;121:1128-1137. [PubMed: 25538019]

38. Clinicaltrials.gov. Stereotactic Body Radiotherapy Dose Escalation in Pancreatic Cancer (SBRT). https://clinicaltrials.gov/ct2/show/NCT02454140 Accessed January 28, 2018.

39. Krishnan S, Chadha AS, Suh Y, et al. Focal Radiation Therapy Dose Escalation Improves Overall Survival in Locally Advanced Pancreatic Cancer Patients Receiving Induction Chemotherapy and Consolidative Chemoradiation. Int J Radiat Oncol Biol Phys 2016;94:755-765. [PubMed: 26972648]

40. Ng S, Herman JM. SBRT for Unresectable Pancreatic Cancer Can Improve Local Control With Minimal Toxicity. Int J Radiat Oncol Biol Phys 2017;99:298-299. [PubMed: 28871974]

41. Phillips TL, Wharam MD, Margolis LW. Modification of radiation injury to normal tissue by chemotherapeutic agents. Cancer 1975;35:1678-1684. [PubMed: 50122]

42. Isaacs RD, Wattie WJ, Wells AU, et al. Massive haemoptysis as a late consequence of pulmonary irradiation. Thorax 1987;42:77-78. [PubMed: 3616975]

43. Langendijk JA, Tjwa MK, de Jong JM, et al. Massive haemoptysis after radiotherapy in inoperable non-small cell lung carcinoma: is endobronchial bracytherapy really a risk factor? Radiother Oncol. 1998;49:175-183. [PubMed: 10052884]

44. Ullrich NJ, Robertson R, Kinnamon DD, et al. Moyamoya following cranial irradiation for primary brain tumors in children, Neurology 2007;68:932. [PubMed: 17372129]

45. Greve J, Bas M, Schuler P, et al. Acute arterial hemorrhage following radiotherapy of oropharyngeal squamous cell carcinoma. Strahlenther Onkol 2010;186:269-273. [PubMed: 20437014] 
46. Yanik B, Keyik B, Conkbayir I, et al. Carotid blowout syndrome with oronasal hemorrhage: magnetic resonance imaging findings. Jpn J Radiol 2011;29:72-75. [PubMed: 21264666]

47. Ito M, Niho S, Nihei K, et al. Risk factors associated with fatal pulmonary hemorrhage in locally advanced non-small cell lung cancer treated with chemoradiotherapy. BMC Cancer 2012;12:27. [PubMed: 22260460]

48. Self EM, Bumpous J, Ziegler C, et al. Risk Factors for Hemorrhage After Chemoradiation for Oropharyngeal Squamous Cell Carcinoma. JAMA Otolaryngol Head Neck Surg 2013;139:356361. [PubMed: 23599070]

49. Aizer AA, Du R, Wen PY, et al. Radiotherapy and death from cerebrovascular disease in patients with primary brain tumors. J Neurooncol 2015;124:291-297. [PubMed: 26080799]

50. El-Fayech C, Haddy N, Allodji RS, et al. Cerebrovascular Diseases in Childhood Cancer Survivors: Role of the Radiation Dose to Willis Circle Arteries. Int J Radiat Oncol Biol Phys 2017;97:278. [PubMed: 28068236]

51. Nordstrom M, Felton E, Sear K, et al. Large Vessel Arteriopathy After Cranial Radiation Therapy in Pediatric Brain Tumor Survivors, J Child Neurol 2018;33:359. [PubMed: 29575995]

52. Elhammali A, Patel M, Weinberg B, et al. Late gastrointestinal tissue effects after hypofractionated radiation therapy of the pancreas. Radiat Oncol 2015;10:186. [PubMed: 26337917] 


\section{HIGHLIGHTS}

- $\quad$ No studies have assessed SBRT+NFV for borderline/unresectable pancreatic cancer.

- This phase I trial enrolled 46 patients, of which 39 received per-protocol therapy.

- 40 Gy SBRT and $1250 \mathrm{mg}$ BID x5wks NFV was the maximum tolerated dose.

- $\quad$ Six (15\%) patients recurred locally, and median overall survival was 14.4 months.

- Attention to treatment planning may reduce the incidence of late GI bleeding. 


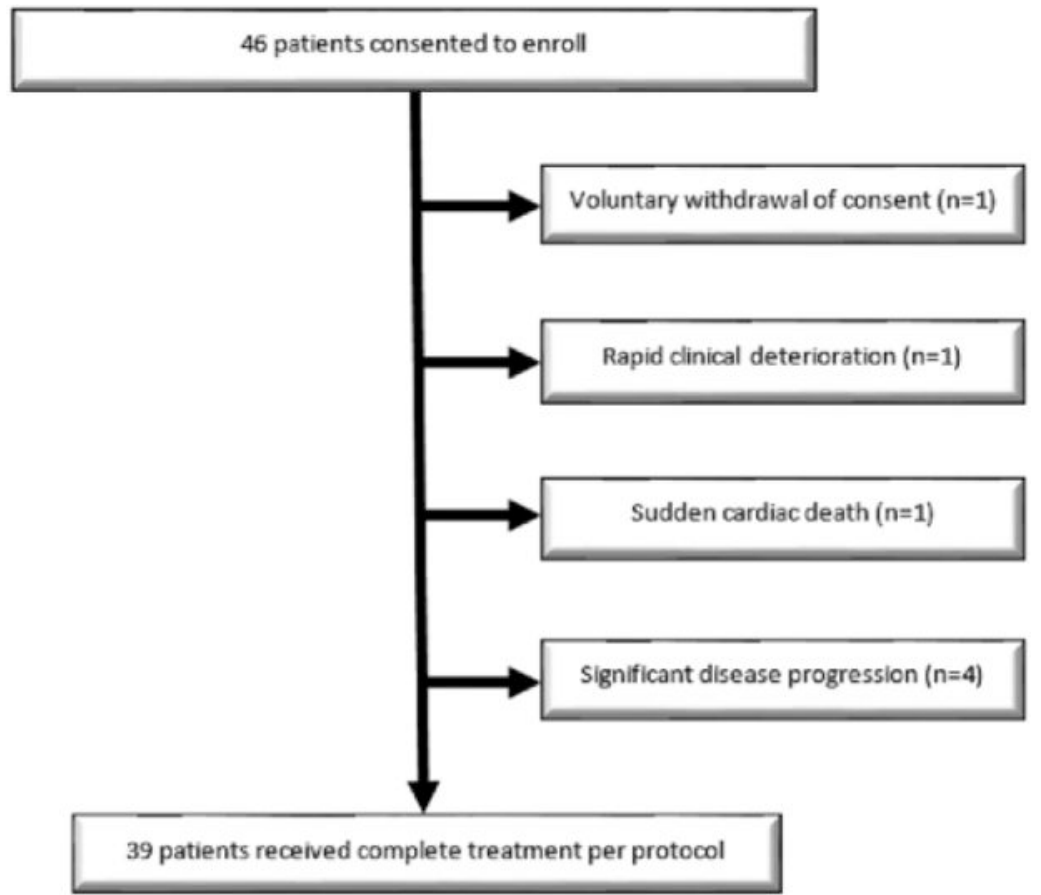

Figure 1.

Study schema. 


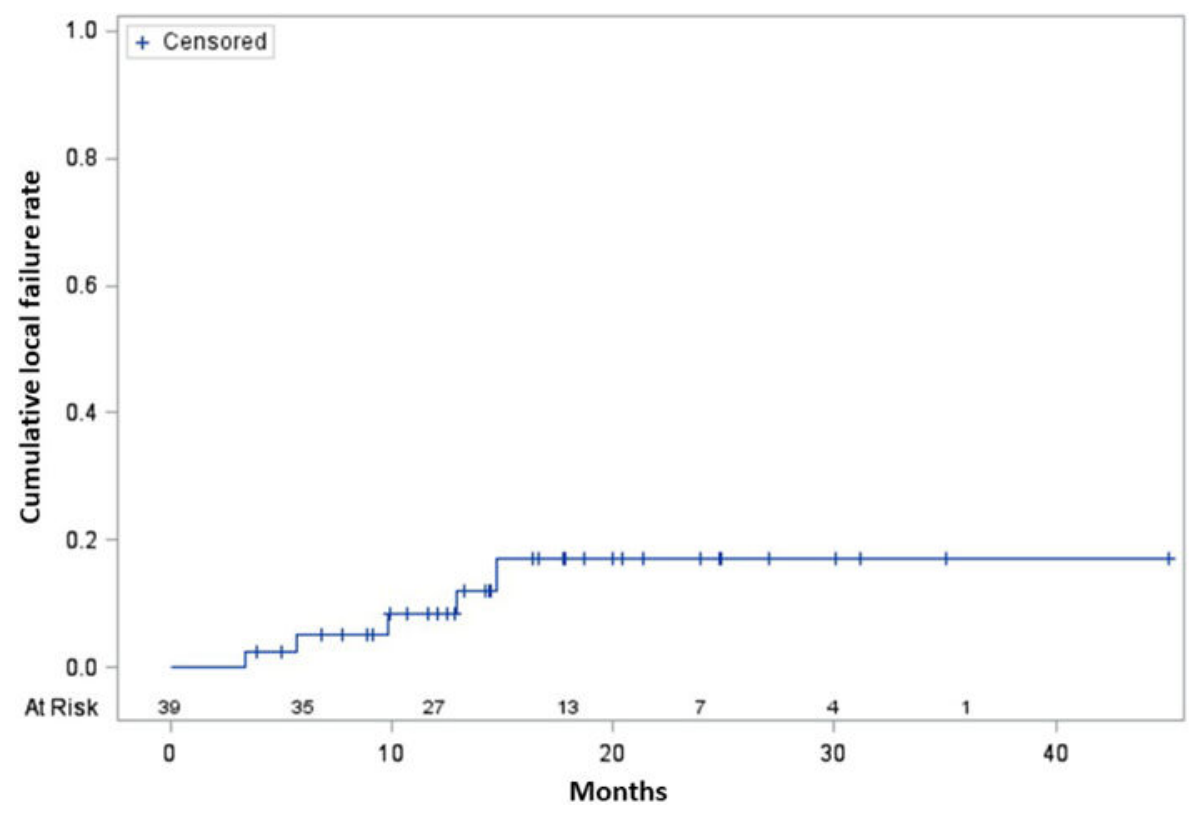



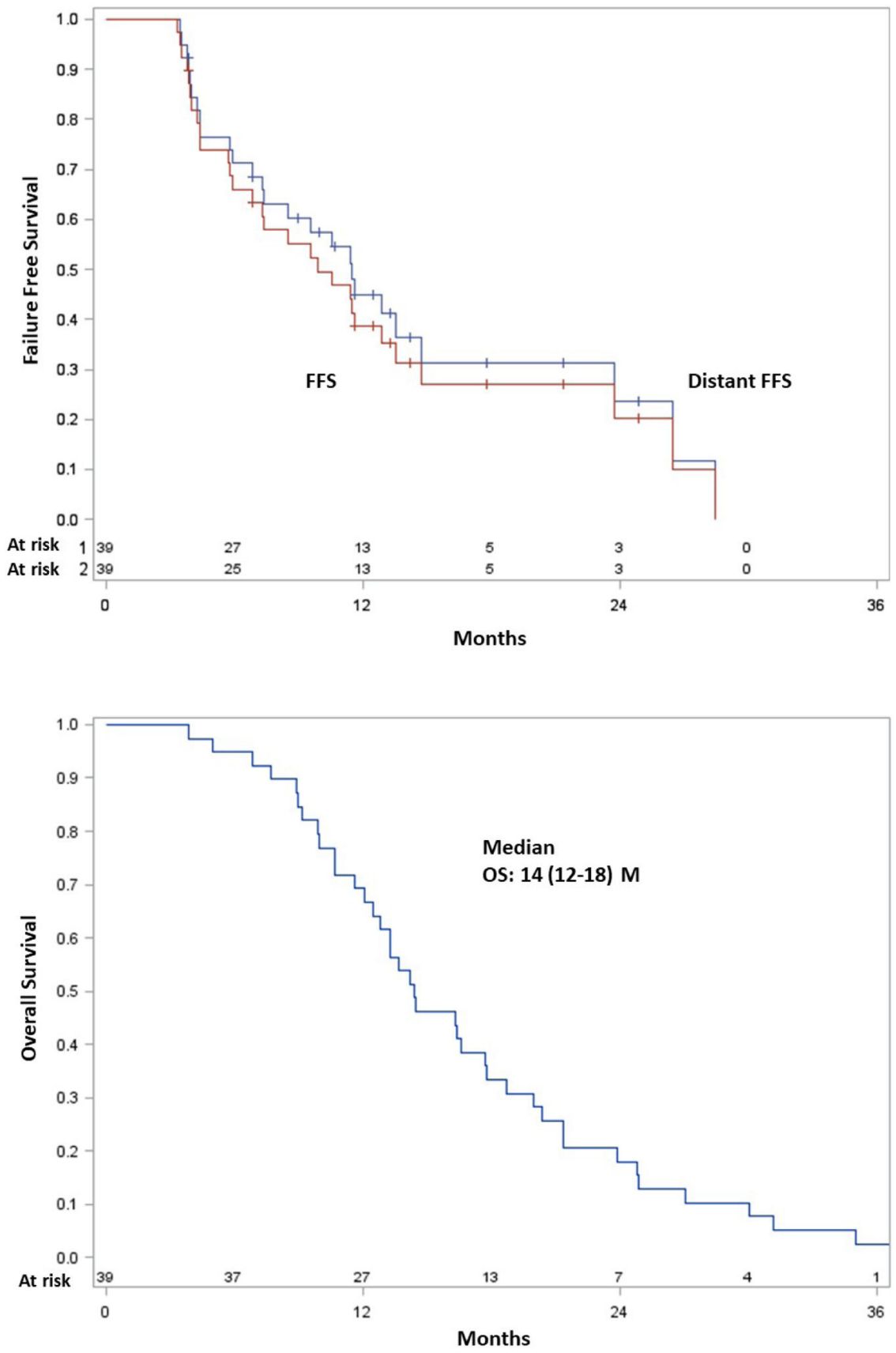

Figure 2.

Cumulative incidence curve of local failures (A), Kaplan-Meier survival curves of distant failure-free survival and (overall) failure-free survival (B), and Kaplan-Meier overall survival curve (C). 

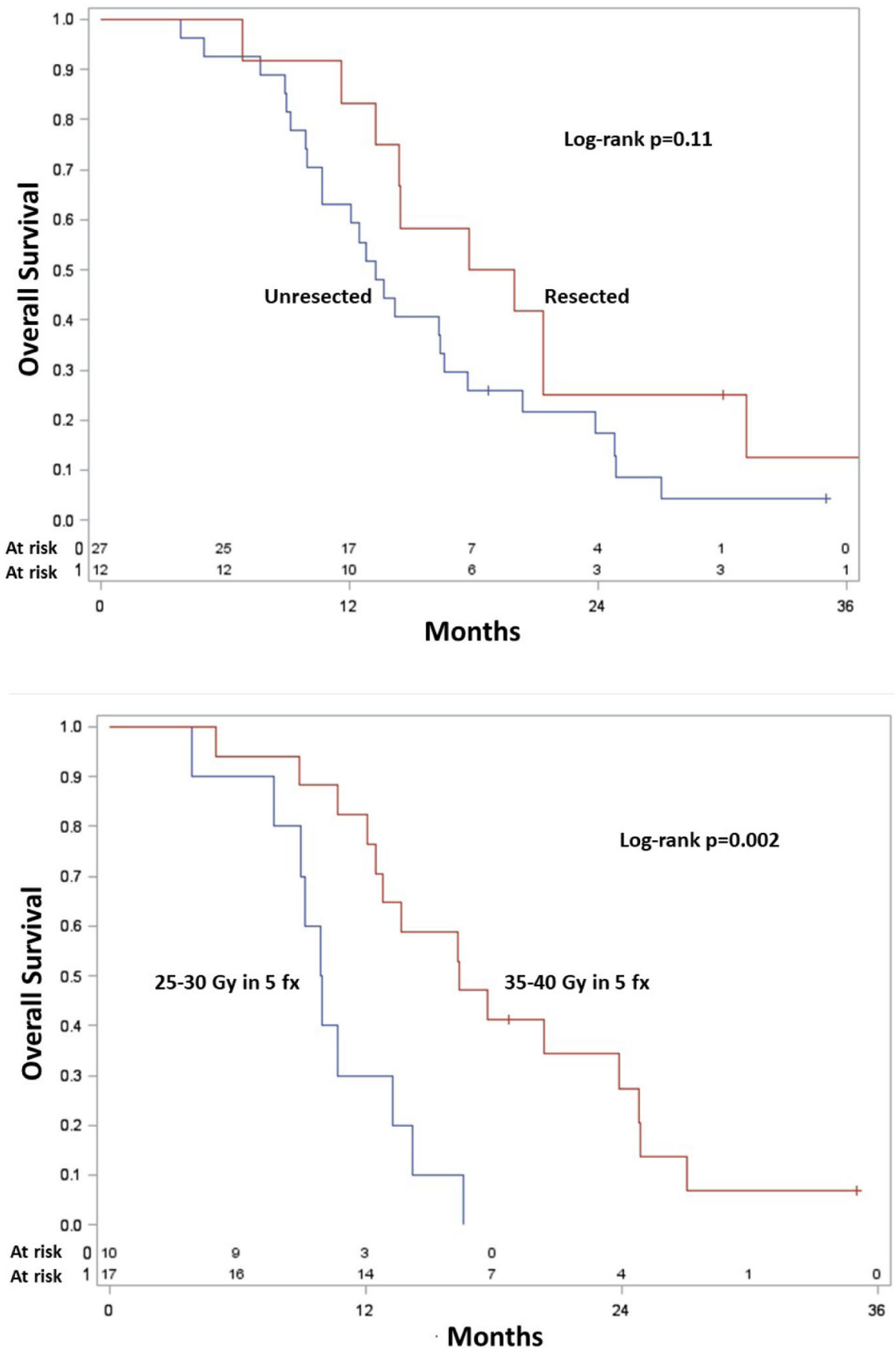

Figure 3.

Kaplan-Meier overall survival curves of patients having undergone surgical resection versus lack thereof (A), and unresected patients having received 7-8 Gy/fraction versus 5-6 Gy/ fraction. 
Table 1.

Selected clinical characteristics of the study population.

\begin{tabular}{|c|c|}
\hline Parameter & $\begin{array}{l}\text { Count } \\
\text { (Percent) }\end{array}$ \\
\hline \multicolumn{2}{|l|}{ Age (years) } \\
\hline Median (range) & $63(35-80)$ \\
\hline \multicolumn{2}{|l|}{ Gender } \\
\hline Male & $23(59 \%)$ \\
\hline Female & $16(41 \%)$ \\
\hline \multicolumn{2}{|l|}{ Race } \\
\hline Caucasian & $37(95 \%)$ \\
\hline Other & $2(5 \%)$ \\
\hline \multicolumn{2}{|l|}{ ECOG performance status } \\
\hline 0 & $13(33 \%)$ \\
\hline 1 & $21(54 \%)$ \\
\hline 2 & $5(13 \%)$ \\
\hline \multicolumn{2}{|l|}{ Tumor size $(\mathrm{cm})$} \\
\hline Median (range) & $3.1(0.6-6.5)$ \\
\hline \multicolumn{2}{|l|}{ Initial resectability status } \\
\hline Borderline resectable & $22(56 \%)$ \\
\hline 1) Contact with SMV/PV $>180^{\circ}$ & 6 \\
\hline 2) Contact with SMV/PV $<180^{\circ}$ with irregularity or thrombosis of vein(s) & 4 \\
\hline 3) Contact with IVC & 1 \\
\hline 4) Contact with SMA or CA $\leq 180^{\circ}$ & 4 \\
\hline 5) Contact with CHA/variant arterial anatomy & 7 \\
\hline Unresectable & $17(44 \%)$ \\
\hline 1)Contact with SMA > $180^{\circ}$ & 11 \\
\hline 2)Contact with $\mathrm{CA}>180^{\circ}$ & 6 \\
\hline \multicolumn{2}{|l|}{ Clinical T classification } \\
\hline T3 (extending beyond pancreas but not involving SMA/CA) & $18(54 \%)$ \\
\hline T4 (involving SMA or CA) & $21(46 \%)$ \\
\hline \multicolumn{2}{|l|}{ Clinical N classification } \\
\hline N0 & $22(56 \%)$ \\
\hline N1 & $17(44 \%)$ \\
\hline \multicolumn{2}{|l|}{ PTV size $\left(\mathrm{mm}^{3}\right)$} \\
\hline Median (range) & $125(37-278)$ \\
\hline
\end{tabular}

Abbreviations: ECOG, Eastern Cooperative Oncology Group; SMV, superior mesenteric vein; PV, portal vein; IVC, inferior vena cava; SMA, superior mesenteric artery; CA, celiac artery; CHA, common hepatic artery; PTV, planning target volume 
Table 2.

Instances of toxicities possibly, probably, or definitely attributed to protocol therapy during and 30 days post stereotactic radiotherapy.

\begin{tabular}{|l|c|c|c|}
\hline Adverse Event & Grade 2 & Grade 3 & Grade 4 \\
\hline Abdominal pain & & 1 & \\
\hline Alkaline phosphatase elevation & & 1 & \\
\hline Anemia & $3^{*}$ & 1 & \\
\hline Anorexia & $2^{*}$ & & \\
\hline Constipation & 1 & & \\
\hline Dehydration & 3 & & \\
\hline Deep vein thrombosis-femoral & & $1^{*}$ & \\
\hline Emesis & & 1 & \\
\hline Fatigue & 2 & $1^{*}$ & 1 \\
\hline Fever & 1 & & \\
\hline Hyperbilirubinemia & 1 & & \\
\hline Hypoalbuminemia & $2^{* *}$ & & \\
\hline Hypokalemia & & 1 & \\
\hline Hypotension & 2 & & \\
\hline Infection & 1 & & 1 \\
\hline Leukopenia & 2 & 1 & \\
\hline Nausea & & & \\
\hline Sepsis & & 1 & \\
\hline Thrombocytopenia & & $\mathbf{1 1}$ & \\
\hline Total & & & \\
\hline
\end{tabular}

* Denotes one patient who experienced this adverse event after 30 days following SBRT completion. 
Table 3.

Dosimetric analysis of patients receiving 7-8 Gray per fraction with gastrointestinal bleeding versus lack thereof.

\begin{tabular}{|l|c|c|c|}
\hline \multicolumn{1}{|c|}{$\begin{array}{c}\text { Parameter } \\
\text { Median (range) }\end{array}$} & $\begin{array}{c}\text { Patients without } \\
\text { GI bleeding } \\
(\mathbf{n = 2 3 )}\end{array}$ & $\begin{array}{c}\text { Patients with GI } \\
\text { bleeding (n=4) }\end{array}$ & $\begin{array}{c}\text { p-value } \\
\text { (Mann-Whitney U) }\end{array}$ \\
\hline PTV (cc) & $122(119-157)$ & $138(37-218)$ & 0.891 \\
\hline PTV mean dose (Gy) & $41(38-41)$ & $42(36-45)$ & 0.260 \\
\hline Duodenum (cc) & $72(66-88)$ & $75(66-91)$ & 0.585 \\
\hline Duodenal mean dose (Gy) & $19(18-21)$ & $22(19-26)$ & 0.116 \\
\hline Duodenal V35 (cc) & $7(7-11)$ & $15(7-21)$ & 0.056 \\
\hline Duodenal V30 (cc) & $18(16-21)$ & $25(17-32)$ & 0.101 \\
\hline Duodenal V25 (cc) & $27(25-32)$ & $39(25-51)$ & 0.117 \\
\hline Duodenal V20 (cc) & $39(35-44)$ & $51(34-66)$ & 0.088 \\
\hline Duodenal V15 (cc) & $48(43-53)$ & $57(43-71)$ & 0.128 \\
\hline Duodenal V10 (cc) & $51(46-58)$ & $61(52-72)$ & 0.076 \\
\hline Duodenal V5 (cc) & $55(50-62)$ & $65(57-74)$ & 0.101 \\
\hline
\end{tabular}

Abbreviations: GI, gastrointestinal; PTV, planning target volume; cc, cubic centimeters; Gy, Gray; $\mathrm{V}_{\mathrm{n}}$, volume of organ receiving at least $n$ Gy 